\title{
Signature of Electronic Correlations in the Optical Conductivity of the Doped Semiconductor Si:P
}

\author{
Marco Hering, ${ }^{1,2}$ Marc Scheffler, ${ }^{1}$, 田 Martin Dressel, ${ }^{1,0}$ 团 and Hilbert v. Löhneysen ${ }^{2}$ \\ ${ }^{1}$ 1. Physikalisches Institut, Universität Stuttgart, D-70550 Stuttgart, Germany \\ ${ }^{2}$ Physikalisches Institut, Universität Karlsruhe, D-76128 Karlsruhe, Germany
}

(Dated: February 14, 2020)

\begin{abstract}
Electronic transport in highly doped but still insulating silicon at low temperatures is dominated by hopping between localized states; it serves as a model system of a disordered solid for which the electronic interaction can be investigated. We have studied the frequency-dependent conductivity of phosphorus-doped silicon in the $\mathrm{THz}$ frequency range (30 $\mathrm{GHz}$ to $3 \mathrm{THz}$ ) at low temperatures $T \geq$ $1.8 \mathrm{~K}$. The crossover in the optical conductivity from a linear to a quadratic frequency dependence as predicted by Efros and Shklovskii is observed qualitatively; however, the simple model does not lead to a quantitative agreement. Covering a large range of donor concentration, our temperature- and frequency-dependent investigations reveal that electronic correlation effects between the localized states play an important and complex role at low temperatures. In particular we find a super-linear frequency dependence of the conductivity that highlights the influence of the density of states, i.e. the Coulomb gap, on the optical conductivity. When approaching the metal-to-insulator transition by increasing doping concentration, the dielectric constant and the localization length exhibit critical behavior.
\end{abstract}

\section{INTRODUCTION}

The transition from a semiconductor to a metal upon doping is so basic and important that it seems hard to believe that this crucial issue is far from being understood, in spite of the enormous effort invested over the years $\stackrel{1,2}{2}$ For low concentration, the dopants provide localized states which are dispersed in the semiconductor crystal. When the host contribution to charge transport can be neglected (at low temperature), the system therefore resembles a perfectly disordered solid. At low excitation energies the charge-carrier transport takes place via hopping processes between these spatially randomly distributed states and is described by variable range hopping (VRH). Although the model originally suggested by Mott ${ }^{3.4}$ neglects correlation effects between the localized electronic states, in certain regimes it describes the phenomena of temperature- and frequency-dependent transport quite well. Pollak ${ }^{5}$ and Srinivasan, $\frac{6}{-}$ and later in particular Efros and Shklovskii $(\mathrm{ES})^{7}$ went one step further and included the long-range electron-electron interaction. During the last thirty years, a large number of analytical, numerical, and experimental results have been collected $^{8,9}$ which clarified the importance of Coulomb correlations to some extent. Nevertheless, a complete and consistent picture cannot be drawn at this point.

In a more general view, the disordered localized states can be called electron glass: in this terminology Mott's model describes the Fermi glass 10 of non-interacting particles, whereas the model taking into account Coulomb interactions between the states is commonly referred to as the Coulomb glass $\stackrel{11}{=}$ With decreasing temperature or decreasing electron density, a transition is expected from a temperature variation of the resistivity corresponding to that suggested for a Fermi glass to a behavior as predicted by ES for the Coulomb glass. $\underline{\underline{9}}$ The dc conductivity is influenced by a Coulomb gap $\Delta$ which opens in the density of states around the Fermi edge $E_{F}$ due to the long-range Coulomb interaction. The transition from one behavior to the other shows that at higher excitation energies the effects of Coulomb interaction are negligible and Mott's VRH behavior is recovered.

For the ac conductivity there exist distinct predictions as well. While Mott described the Fermi glass behavior of non-interacting particles, $\stackrel{4}{=} \mathrm{ES}$ supplemented this derivation by introducing the interaction between those two states directly involved in the hopping process that is triggered by resonant absorption of a photon. $\underline{12}$ Thus with increasing frequency there is also a transition from the Coulomb glass regime to the Fermi glass.

Within the model of ES,,$\underline{9}$ the Coulomb gap can be roughly evaluated from the temperature dependence of the dc conductivity in the two regimes. A more direct observation of the Coulomb correlation gap in the density of states is possible by tunnelling spectroscopy 13,14 Quite recently, a controversy arose whether it is really the Coulomb gap which determines the crossover energy scale from the Fermi- to the Coulomb-glass-like behavior in the frequency-dependent transport. According to ES, this transition is driven by the Coulomb energy of the sites forming the resonant pair. However, Lee and Stutzmann $\frac{15}{15}$ claimed instead that the transition occurs when the photon energy equals twice the Coulomb gap width. A subsequent experimental study $\underline{\underline{16}}$ and computer simulations ${ }^{17}$ seem to support the former view. It was recently suggested 18 that many-particle correlations might be crucial for a quantitative description of the hopping conductivity. Here we try to resolve this issue by providing more accurate data in a wider range of carrier concentration and a broader range of frequency.

A prime example to study the physics of disordered solids is phosphorus-doped silicon which can be tuned in the insulating regime as well as through the metalinsulator transition (MIT) at a critical concentration 
$n_{c}=3.5 \cdot 10^{18} \mathrm{~cm}^{-3}$ by varying the donor concentration $n$ in the single-crystalline host $1,19,20$ When going to low enough temperatures and frequencies, the crossover from a Fermi glass to a Coulomb glass can nicely be investigated in $\mathrm{Si}: \mathrm{P} 21$ Other options to tune the system are the application of magnetic field ${ }^{22,23}$ and pressure $\frac{24,25}{25}$ as has also been done for Si:B $\stackrel{26}{26}$ For small enough doping concentration $n<2.78 \cdot 10^{18} \mathrm{~cm}^{-3}$, a different effect was inferred by the strong $\rho(T)$ dependence; the VRH subsides and a simple activated behavior is observed $\frac{1,21}{\underline{E}}$ Due to on-site Coulomb repulsion, a Hubbard gap $E_{2}$ splits the impurity band. While a clear distinction between uncompensated $\mathrm{Si}: \mathrm{P}$ and compensated $\mathrm{Si}:(\mathrm{P}, \mathrm{B})$ is seen in dc transport, $\stackrel{27}{\longrightarrow}$ it is not clear how this effects the frequency-dependent transport.

The work presented in the following mainly concentrates on the temperature and frequency variation of the conductivity; the experimental data are compared with the theory by Shklovskii and Efros in order to elucidate the influence of electronic correlations on the transport in disordered electron solids. In addition, the measurements on samples with various doping concentrations give insights into the scaling behavior at the MIT.

\section{THEORETICAL CONSIDERATIONS}

Before we describe our experimental results, we briefly present the relevant theories and phenomenological models. More details and discussion can be found in well established monographs and reviews $\underline{4.9,28}$

\section{A. Fermi glass}

Following Mott's theory of the Fermi glass, $\frac{4}{,}$ the electrical dc conductivity $\sigma_{d c}$ as a function of temperature $T$ for a non-interacting three-dimensional electron glass (variable range hopping) is given by the well-known $T^{-1 / 4}$ law

$$
\sigma_{d c}(T) \propto \exp \left\{-\left(T_{\mathrm{Mott}} / T\right)^{1 / 4}\right\}
$$

with a characteristic temperature

$$
T_{\mathrm{Mott}}=21.2 \frac{1}{k_{B} N_{0}\left(E_{F}\right) \xi^{3}}
$$

Here $\xi$ is the localization length and $N_{0}$ is the density of electronic states (DOS) at the Fermi level $E_{F}$ which is assumed to be constant close to $E_{F}$ in the absence of electronic correlations. The Mott temperature $T_{\mathrm{Mott}}$ tends to zero as the MIT is approached ${ }^{21}$

The common theoretical approach to the ac transport in statistically disordered solids is the so-called pair approximation. Each photon-induced hopping process between two localized states has a certain probability. The sum over all the hops contributing to the absorption process leads to the photon-assisted conductivity of the system. In the derivation of the ac conductivity, a simple one-electron model of the disordered system is assumed, neglecting any correlation effects between the states $\stackrel{4}{=}$ Apart from logarithmic corrections, the frequency-dependent $(\omega=2 \pi f)$ conductivity of a Fermi glass at $T=0$ shows a quadratic behavior:

$$
\sigma_{1}(\omega)=\alpha e^{2} N_{0}^{2} \hbar \omega^{2} \xi r_{\omega}^{4} .
$$

Here we denote by $\sigma_{1}$ the real part of the complex conductivity $\hat{\sigma}=\sigma_{1}+i \sigma_{2}$; the imaginary part is related to the real part of the dielectric constant $\epsilon_{1}=1-4 \pi \sigma_{2} / \omega \cdot \underline{29}$ The length $r_{\omega}=\xi \cdot \ln \left\{2 I_{0} / \hbar \omega\right\}$ is the most probable distance for the hopping processes. Here $I_{0}$ is the prefactor describing the overlap integral; its value is of the order of the binding energy of the electronic state,, 28 and hence it is commonly taken to be the Rydberg energy of the dopant (for Si:P we use $I_{0} \approx 45 \mathrm{meV}$ ). The factor $\alpha$ is a constant of order of unity.

\section{B. Coulomb glass}

In the case of appreciable (long-range) electronic interaction, the DOS approaching the Fermi energy is reduced due to the Coulomb interaction between the localized states, leading to $N(E) \approx(3 / \pi)\left(E-E_{F}\right)^{2} \epsilon_{1}^{3} / e^{6}$. The width of this so-called Coulomb gap is given by

$$
\Delta \propto e^{3} N_{0}^{1 / 2} / \epsilon_{1}^{3 / 2} .
$$

This concept was confirmed by tunnelling experiments 13,14 which reveal a parabolic dependence of the conductance measuring the DOS. The typical size of the Coulomb gap in doped semiconductors is $1 \mathrm{meV}$. Taking the Coulomb gap into account, Shklovskii and Efros ${ }^{9}$ calculated the temperature dependence of the dc conductivity, the so-called $T^{-1 / 2}$ law

$$
\sigma_{d c}(T) \propto \exp \left\{-\left(T_{\mathrm{ES}} / T\right)^{1 / 2}\right\}
$$

with the characteristic temperature

$$
T_{\mathrm{ES}}=2.8 \frac{e^{2}}{k_{B} \epsilon_{1} \xi},
$$

which is a measure of the characteristic Hartree interaction strength. At large enough $T$ the accessible energy range for hopping processes, $k_{B}\left(T^{3} T_{\mathrm{Mott}}\right)^{1 / 4}$, exceeds the Coulomb gap $\Delta$, and Mott's VRH behavior Eq. (1) is recovered. This crossover from a Fermi glass to a Coulomb glass can be seen as a weak kink in the temperaturedependent conductivity $\underline{\underline{21}}$

The ac conductivity is again calculated in the pair approximation. When taking into account the average Coulomb attraction $U\left(r_{\omega}\right)=e^{2} / \epsilon_{1} r_{\omega}$ between two sites forming a resonant pair of distance $r_{\omega}$, hopping processes can even occur between states that are separated energetically by more than the photon excitation energy $\hbar \omega$. 
This long-range Coulomb interaction $U\left(r_{\omega}\right)$ strongly depends on the real part of the dielectric constant $\epsilon_{1}$. As will be seen later, $\epsilon_{1}$ diverges as the MIT is approached; it basically screens the Coulomb interaction. The resulting optical conductivity

$$
\sigma_{1}(\omega)=\alpha e^{2} N_{0}^{2} \omega \xi r_{\omega}^{4}\left[\hbar \omega+U\left(r_{\omega}\right)\right]
$$

is distinct from Eq. (3) for small excitation energies $\hbar \omega \ll U\left(r_{\omega}\right)$, when the frequency-dependent conductivity yields approximately a linear behavior:

$$
\sigma_{1}(\omega)=\frac{\alpha e^{4}}{\epsilon_{1}} N_{0}^{2} \hbar \omega \xi^{4}\left[\ln \left\{2 I_{0} / \hbar \omega\right\}\right]^{3} .
$$

In the opposite case of weak correlations, $\hbar \omega \gg U\left(r_{\omega}\right)$, the frequency dependence of Eq. (7) is quadratic if the logarithmic corrections are neglected. Naturally, it corresponds to Mott's formula Eq. (3). Hence the transition from linear to quadratic behavior can be considered as a transition from probing the interacting Coulomb glass at low frequency to the Fermi glass regime at high frequency where correlation effects can be neglected.

Although for both the temperature-dependent dc data and the low-temperature ac behavior a crossover is predicted, the canonical theory presented so far does not represent a direct correspondence. In the former case the reduced DOS due to Coulomb interaction - the Coulomb gap - modifies Mott's $T^{-1 / 4}$ law to the ES $T^{-1 / 2}$ law. In the latter case, however, Eq. (7) and Eq. (8) have been calculated under the assumption of a constant DOS at the Fermi level although meant to describe the interacting system. Since the DOS of a Coulomb glass exhibits the Coulomb gap of width $\Delta$, this assumption is valid only for $U\left(r_{\omega}\right) \gg \Delta$, i.e. when the Coulomb interaction influences the occupation numbers only to a small extent. Then only states outside the gap contribute to $\sigma_{1}(\omega)$.

In the opposite case, mainly states within the gap participate in the hopping conduction. For $\Delta>U\left(r_{\omega}\right)>$ $\hbar \omega$, Shklovskii and Efros ${ }^{12}$ derive a stronger frequency variation than given by Eq. (8). Hence, the incorporation of the Coulomb gap results in a super-linear frequency dependence

$$
\sigma_{1}(\omega)=\frac{\alpha e^{4}}{\epsilon_{1}} N_{0}^{2} \xi^{4} \frac{\hbar \omega}{\ln \left\{2 I_{0} / \hbar \omega\right\}}
$$

in contrast to the sub-linear dependence of Eq. (8) for constant DOS. Between both limiting cases a smooth crossover is expected. Computer simulations 17 yield larger exponents and a more abrupt transition compared to the predictions of $\mathrm{ES} .9 .12$

In principle, frequency-dependent measurements provide the possibility to investigate electron glasses like Si:P in the relevant energy scales. But due to experimental difficulties to access the very low energies required $(\mathrm{meV}$ and less, corresponding to the $\mathrm{GHz}$ and $\mathrm{THz}$ frequency range), the expected crossover from Coulomb glass to Fermi glass has been observed only recently, $\stackrel{15,16}{=}$ At this point it is not clear how the opening of a Hubbard gap in the density of states influences the frequency dependent conductivity.

\section{Metal-insulator transition}

So far we have only discussed the insulating state of disordered systems. In our case the doping of the host silicon crystal adds an extra dimension as we approach the MIT with increasing doping. This quantum phase transition from an insulator with localized states and zero dc conductivity at $T=0$ to a metal with extended electronic states associated with finite dc conductivity can be driven by varying external parameters like uniaxial stress, magnetic fields or, in our case, the doping concentration $n \underline{1,20,30,31}$ The MIT in doped semiconductors like Si:P is due to two facts: the localization arising from disorder (Anderson transition) and from long-range electronelectron interaction due to the loss of screening as states become localized (Mott-Hubbard transition), 1,30,32 The spatial extent of the electronic states scales with doping; the localization length increases as the MIT is approached

$$
\xi \propto\left|n_{c}-n\right|^{-\nu}
$$

where $n_{c}$ is the critical concentration $9.33,34,35$ On the metallic side also the conductivity scales: $: \frac{36,37}{2} \sigma_{1} \propto$ $\left|n_{c}-n\right|^{\mu}$. Theoretically, $\mu$ is inferred from the correlationlength critical exponent $\mu=(d-2) \nu$ by scaling arguments $; \stackrel{34}{=} d$ indicates the dimensionality of the system. Also the dielectric constant diverges as ${ }^{37,38}$

$$
\epsilon_{1} \propto\left|n_{c}-n\right|^{-\zeta^{\prime}} .
$$

The power laws are supposed to be universal; that is the critical exponents do not depend on the details of the geometric structure or the interaction. Previous investigations of uncompensated semiconductors inferred an exponent $\mu=0.5$, in contrast to $\mu=1$ found for compensated semiconductors and amorphous metals 20 However, it turned out that the exponent depends on the parameter by which the MIT is tuned and on how close to the MIT the experiments are performed. $\underline{\underline{1}}$ Numerical simulations and experiments on metal-insulator composites give values for the critical exponent that scatter considerably, for instance $\zeta^{\prime}$ between 0.5 and $1,39,40,41,42$ In three dimensions, the relation $\zeta^{\prime}=2 \mu$ is expected. ${ }^{43}$ McMillan $\underline{44}$ suggested that both critical exponents are related by $\zeta^{\prime}=\nu(\eta-1)$ where $1<\eta<3$. Applied to our case, it is not obvious that the crucial assumption of this model really holds that only states within the Coulomb gap are of relevance. Recent computer calculations support this scepticism: 17

The above considerations neglect the on-site Coulomb interaction: For uncompensated semiconductors like $\mathrm{Si}: \mathrm{P}$, a twofold spin-degenerate ground state $1 \mathrm{~s}\left(\mathrm{~A}_{1}\right)$ (and 
higher valley-orbit split states not considered here) is induced with each $\mathrm{P}$ donor atom. Hence uncompensated $\mathrm{Si}: \mathrm{P}$ is always at half-filling of the impurity band; only the lower Hubbard band is occupied. With increasing $n$ the two Hubbard bands start overlapping at $n_{0}=0.8 n_{c}^{1,27}$ but states at the edges are still Anderson localized. It is only at $n_{c}$ that they become extended.

\section{EXPERIMENTS AND RESULTS}

The samples for this study were cut from two different Czochralski-grown, nominally uncompensated silicon single crystals with a phosphorus gradient along the growth axis. ${ }^{45}$ From the ingots a number of disks were prepared using a diamond wire saw. To remove distorted surface layers, $\frac{47}{4}$ the crystals were chemically and mechanically treated by well established procedures. According to Thurber et $a l l^{48}$ the doping concentration was determined from the room-temperature resistivity employing a commercial four probe measurement system (FPP 5000 by Veeco Instruments). For high doping levels the resistivity ratio $\rho(4.2 \mathrm{~K}) / \rho(300 \mathrm{~K})$ (determined from standard dc measurements) is consistent with Ref. 49. The dopant concentration of the crystals used in this study ranges between $0.89 \cdot 10^{18} \mathrm{~cm}^{-3}$ and $3.04 \cdot 10^{18} \mathrm{~cm}^{-3}$; the sample properties are summarized in Table 1

For optical experiments specimens of $10 \times 10 \mathrm{~mm}^{2}$ were prepared with different thickness ranging from $2 \mathrm{~mm}$ to $0.05 \mathrm{~mm}$ and less. The crystals were chemically and mechanically polished to optical quality. In combination with highly parallel opposite faces, this ensures pronounced Fabry-Perot resonances. Using a quasi-optical Mach-Zehnder interferometer in the $\mathrm{THz}$ range of frequency equipped with backward-wave oscillators as coherent and tunable radiation sources,,$\frac{50}{5}$ the optical transmission and change in phase could be measured between $30 \mathrm{GHz}$ and $1.2 \mathrm{THz}$, corresponding to an energy range between $0.1 \mathrm{meV}$ and $5 \mathrm{meV}$. The Fabry-Perot resonances due to multi-reflection at the surfaces of the silicon sample were utilized to enhance the sensitivity and accuracy 51 From the spectra the real parts of the conductivity $\sigma_{1}(\omega)$ and the dielectric constant $\epsilon_{1}(\omega)$ can be calculated using the Fresnel formula 29 with an uncertainty of $10 \%$ and $5 \%$, respectively. Complementary optical experiments were performed using a Fourier transform spectrometer (modified Bruker IFS 113v) up to frequencies of $3 \mathrm{THz}$ (corresponding to $12 \mathrm{meV}$ ). Depending on the dopant concentration, two to four different thicknesses were measured of each sample in order to optimize the sensitivity. For the sample with phosphorus concentration $n=0.89 \cdot 10^{18} \mathrm{~cm}^{-3}$, the absorption is too weak to determine the conductivity in the entire frequency range. On the other hand, highly conducting specimens with $n \geq 2.9 \cdot 10^{18} \mathrm{~cm}^{-3}$ do not transmit sufficiently to achieve a signal-to-noise ratio large enough to analyze the data with respect to $\sigma_{1}$ over the whole accessible frequency range.

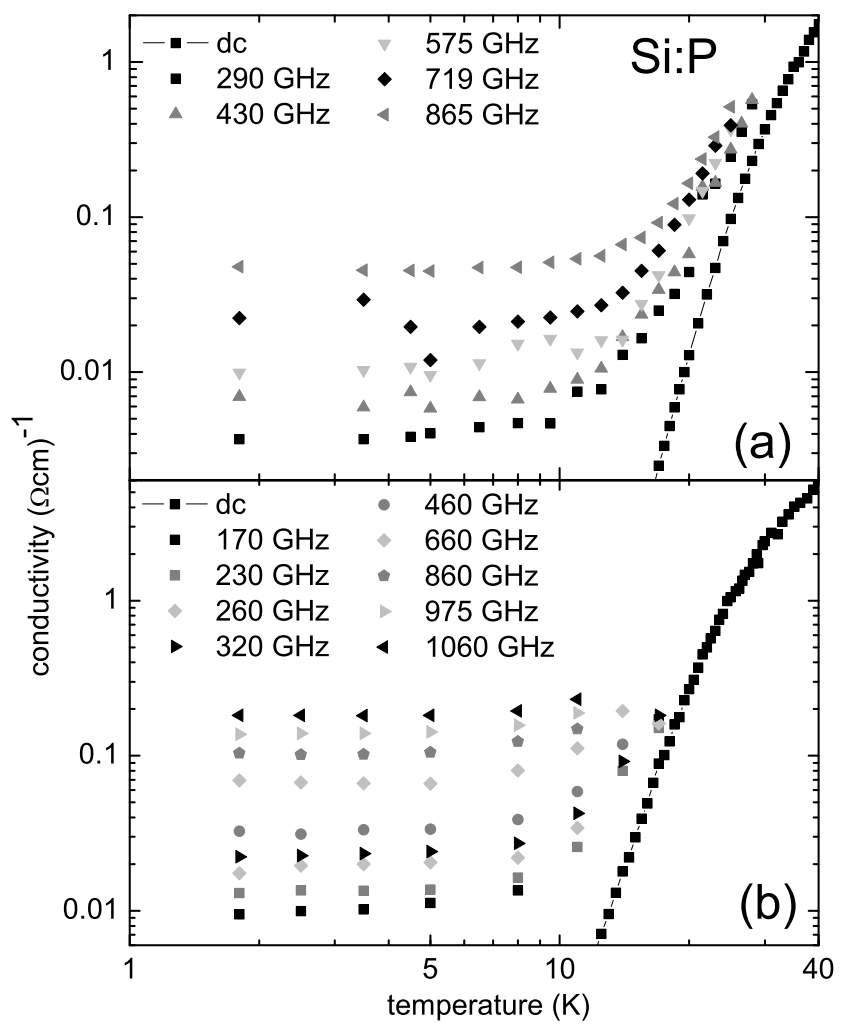

FIG. 1: Temperature dependence of the real part of the conductivity $\sigma_{1}$ for various frequencies and the temperature dependent dc results for Si:P crystals with (a) $n=1.6 \cdot 10^{18} \mathrm{~cm}^{-3}$ and (b) $n=1.97 \cdot 10^{18} \mathrm{~cm}^{-3}$.

Temperatures down to $1.8 \mathrm{~K}$ were reached with a pumped liquid ${ }^{4} \mathrm{He}$ optical cryostat. As an example, in Fig. 1(a) the temperature dependence of the conductivity as obtained from optical measurements is displayed for a sample with $n=1.6 \cdot 10^{18} \mathrm{~cm}^{-3}$. The ac conductivity curves below approximately $10 \mathrm{~K}$ suggest that phononinduced charge-carrier transport can be neglected, and thus the system is in the so-called zero-phonon regime; in other words $\hbar \omega>k_{B} T$. This is a basic requirement in order to compare the measured conductivity spectra $\sigma(\omega)$ with the theoretical predictions discussed above. Because phonon-induced processes dominate at higher temperatures, the ac conductivity curves and the dc data coincide. Obviously, the higher the frequency of a particular curve, the higher the temperature at which this occurs. Similar behavior is observed for the other samples. Fig. 1(b) shows the data for a crystal with higher phosphorus concentration $n=1.97 \cdot 10^{18} \mathrm{~cm}^{-3}$.

\section{ANALYSIS AND DISCUSSION}

\section{A. AC Conductivity}

From the frequency-dependent transmission measurements at various temperatures, the optical conductivity 
TABLE I: List of Si:P crystals used in this study. The phosphorus concentration is indicated by $n$; also the ratio with respect to the critical concentration $n_{c}=3.5 \cdot 10^{18} \mathrm{~cm}^{-3}$ is given. $\rho(300 \mathrm{~K})$ is the dc resistivity measured at room temperature; the low-temperature to high-temperature resistivity ratio is indicated by $\rho(4.2 \mathrm{~K}) / \rho(300 \mathrm{~K})$. The crossover frequency $\omega_{c 1} / 2 \pi$ is obtained by the intersection of the linear and quadratic fits of the two regimes. If the frequency-dependent conductivity is fitted by power laws $\sigma_{1}(\omega) \propto \omega^{s}$, the exponents $s_{\mathrm{CG}}$ and $s_{\mathrm{FG}}$ are obtained for Coulomb glass and Fermi glass, respectively. These fits give the approximate crossover frequency $\omega_{c 2} / 2 \pi$ between both regimes. For samples marked $\triangleright(\triangleleft)$ the conductivity $\sigma_{1}$ could not be determined from Fabry-Perot resonances because the absorption is too high (low). For sample No. 5 only the dielectric constant was measured at low temperatures but not the conductivity (denoted by - ). $T_{0}$ is the temperature of the minimum in the dielectric constant. $\epsilon_{1}$ corresponds to the dielectric constant measured at $T=1.8 \mathrm{~K}$ in the THz range of frequency; $\chi$ is the dielectric susceptibility.

\begin{tabular}{|c|cc|cc|c|ccc|cc|c|}
\hline No. & $\begin{array}{c}n \\
\left(10^{18} \mathrm{~cm}^{-3}\right)\end{array}$ & $n / n_{c}$ & $\begin{array}{c}\rho(300 \mathrm{~K}) \\
(\Omega \mathrm{cm})\end{array}$ & $\frac{\rho(4.2 \mathrm{~K})}{\rho(300 \mathrm{~K})}$ & $\begin{array}{c}\omega_{c 1} / 2 \pi \\
(\mathrm{GHz})\end{array}$ & $\begin{array}{r}s_{\mathrm{CG}} \\
s_{\mathrm{FG}}\end{array} \begin{array}{r}\omega_{c 2} / 2 \pi \\
(\mathrm{GHz})\end{array}$ & $\begin{array}{l}T_{0} \\
(\mathrm{~K})\end{array}$ & $\epsilon_{1}$ & $4 \pi \chi$ \\
\hline 1 & 0.89 & 0.25 & 0.0245 & $4.4 \cdot 10^{10}$ & $\triangleleft$ & $\triangleleft$ & $\triangleleft$ & $\triangleleft$ & 11 & 12.90 & 1.20 \\
2 & 1.60 & 0.46 & 0.0180 & $1.48 \cdot 10^{8}$ & 540 & 1.16 & 2.15 & 760 & 9 & 15.51 & 3.81 \\
3 & 1.97 & 0.56 & 0.0162 & $1.53 \cdot 10^{7}$ & 460 & 1.21 & 2.16 & 550 & 8 & 18.13 & 6.43 \\
4 & 2.29 & 0.65 & 0.0149 & $3.49 \cdot 10^{6}$ & 480 & 1.17 & 2.25 & 630 & 6 & 20.47 & 8.77 \\
5 & 2.40 & 0.69 & 0.0145 & - & - & - & - & - & 6 & 23.56 & 11.86 \\
6 & 2.57 & 0.73 & 0.0139 & $3.46 \cdot 10^{4}$ & 440 & 1.33 & 2.28 & 570 & 5 & 26.48 & 14.78 \\
7 & 2.91 & 0.83 & 0.0130 & 1060 & $\triangleright$ & $\triangleright$ & $\triangleright$ & $\triangleright$ & 5 & 41.57 & 29.87 \\
8 & 3.04 & 0.87 & 0.0127 & 80 & $\triangleright$ & $\triangleright$ & $\triangleright$ & $\triangleright$ & & 55.45 & 43.75 \\
\hline
\end{tabular}

and dielectric constant could be evaluated for samples No. 2 through 6 as described above. This covers the doping range from $1.6 \cdot 10^{18} \mathrm{~cm}^{-3}$ to $2.57 \cdot 10^{18} \mathrm{~cm}^{-3}$. Here we confine ourselves to the lowest-temperature data $(T=1.8 \mathrm{~K})$ in order to ascertain that the experiments are performed in the zero-phonon regime $\left(k_{B} T<\hbar \omega\right)$ for all frequencies of interest. In Fig. 2 the optical conductivity for the sample with doping concentration $n=1.6 \cdot 10^{18} \mathrm{~cm}^{-3}$ is presented. The data can be well described by a crossover from a linear to a quadratic behavior of $\sigma_{1}(\omega)$ as indicated by the solid lines. As pointed out in Sec. [II, this corresponds to the limiting cases of a Fermi glass with no interaction at high frequencies and a Coulomb glass with electronic correlations at low frequencies, respectively. The corresponding crossover frequencies $\omega_{\mathrm{c} 1}$ are listed in Tab. [1

The dashed line in Fig. 2 is drawn according to the quantitative description given in Eq. (7); obviously the experimentally observed crossover is much more pronounced than the smooth transition predicted by the theory of Shklovskii and Efros $\underline{\underline{8}, \underline{9}, 12}$ This strong discrepancy of theory and experiment has already been observed by previous investigations $\frac{15,16}{}$

Upon closer inspection as presented in Fig. 3, the lowfrequency data of all samples are better described by a super-linear frequency dependence as suggested by ES in Eq. (9) than by a purely linear behavior. (Note that the logarithmic corrections in Eq. (8) even lead to a sublinear frequency dependence.) The logarithmic corrections $\sigma_{1}(\omega) \propto \omega / \ln \left\{2 I_{0} / \hbar \omega\right\}$ for Coulomb glasses have to be taken into account if the Coulomb interaction energy is smaller than the width of the Coulomb gap, $U\left(r_{\omega}\right)<\Delta$. The resulting fits are shown for two samples in Fig. 3 , with the exactly linear fits for comparison.

In the opposite limit of high frequencies where the pre- dictions for a Fermi glass apply, a slightly stronger frequency variation is recovered than expected from Eq. (7). A fit by a power-law $\sigma(\omega) \propto \omega^{s}$ yields the exponent $s$. As seen in Tab. I $s(n)$ increases with doping concentration from $s=2.15$ for $n=1.6 \cdot 10^{18} \mathrm{~cm}^{-3}$ to $s=2.28$ for $n=2.57 \cdot 10^{18} \mathrm{~cm}^{-3}$. An overview of the spectra of samples with different phosphorus concentration is given in Fig. 4t together with the corresponding fit of the superlinear behavior at low frequencies and the approximately quadratic dependence at higher frequencies.

We may compare our findings to previous investigations deep in the Coulomb glass state. In the radiofrequency range $\left(10^{2}\right.$ to $10^{5} \mathrm{~Hz}$ ) at $T=13 \mathrm{mK}$ (in a sam-

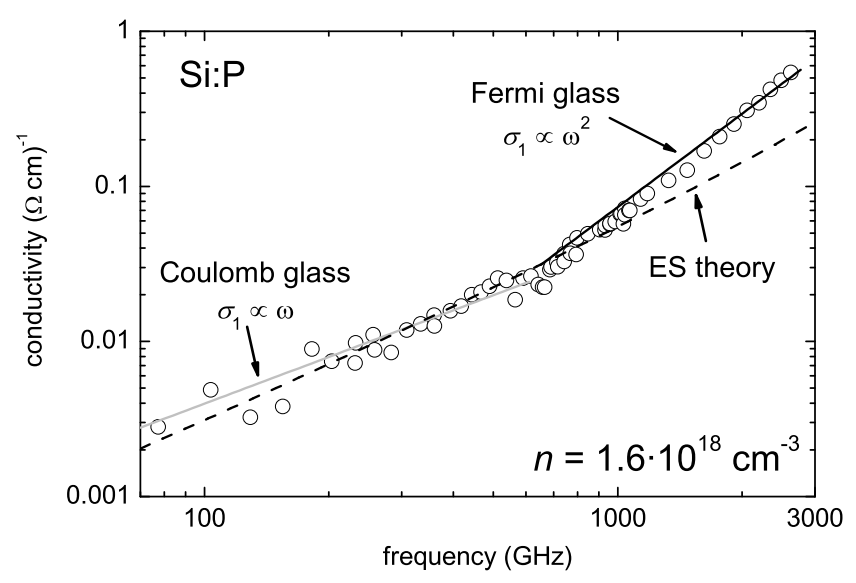

FIG. 2: Frequency dependence of a Si:P sample with $n=$ $1.6 \cdot 10^{18} \mathrm{~cm}^{-3}$ at $T=1.8 \mathrm{~K}$. The straight lines are fits with linear and quadratic behavior respectively. The dashed line is a fit with the prediction of Efros and Shklovskii ${ }^{9}$ given in Eq. (7). The error bars are of the size of the symbols. 


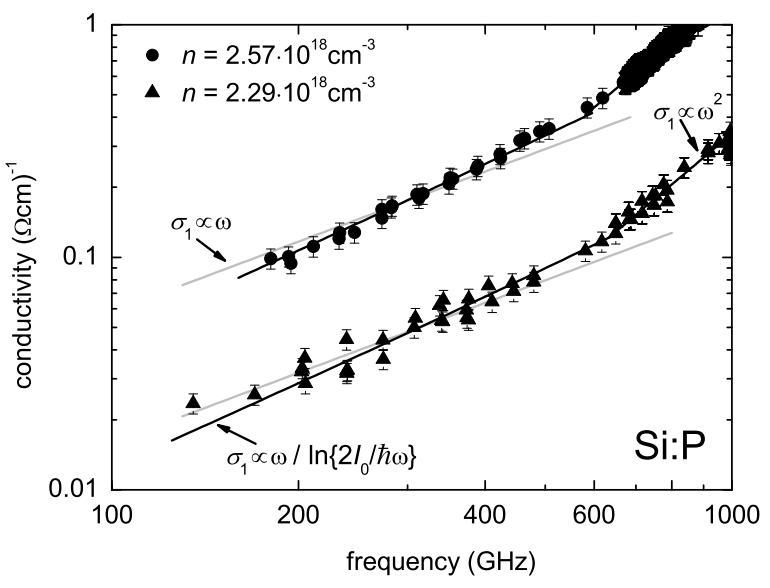

FIG. 3: Frequency-dependent conductivity in the Coulomb glass regime for the silicon samples with phosphorus concentration $n=2.29 \cdot 10^{18} \mathrm{~cm}^{-3}$ and $n=2.57 \cdot 10^{18} \mathrm{~cm}^{-3}$. The fits correspond to a super-linear behavior as indicated in the graph with $I_{0}=45 \mathrm{meV}$. At higher frequencies, the transition to quadratic behavior is apparent. Linear behavior is plotted as gray lines for comparison.

ple close to the MIT and under uniaxial stress) Paalanen et $a l \stackrel{24}{=}$ found $\sigma_{1}(\omega) \propto \omega^{s}$ with $s=0.9$ in Si:P. Castner and coworkers investigated Si:As with $\omega / 2 \pi<2 \mathrm{GHz}$ for $T>1 \mathrm{~K}$ and found a super-linear frequency dependence with $1<s<1.5$ and a very strong doping dependence of $s$ which was interpreted as possible qualitative agreement with Mott's $\omega^{2}$ law $\frac{52}{2}$ For Si:P they observed an increase of $s(n)$ from about unity to 2.2 with increasing $n$ in a density range comparable to our samples $\underline{53}$ Based on previous experiments, $\stackrel{54}{=}$ Castner ${ }^{28}$ argues that this might be due to a decrease in the Coulomb energy $U$ as the MIT is approached. In the light of our results, the following interpretation seems plausible: the observed exponents for Si:As correspond to the super-linear behavior in the Coulomb gap regime, and for Si:P the concentration range might include the transition from Coulombto Fermi-glass behavior but was not identified because of the limited number of frequencies in a comparably small frequency range.

As already pointed out above, the transition between the two regimes is clearly seen and much more abrupt than expected from the general description in Eq. (7) suggested by ES. From the intersection of the fit curves to power-laws, a crossover energy $\hbar \omega_{c 2}$ can be defined. The values obtained by this procedure are summarized in Tab. II. It should be noted that the resulting crossover frequencies $\omega_{c 2}$ are larger than the values $\omega_{c 1}$ obtained from the intersection of linear and quadratic fits (also listed in Tab. (1).

The crossover frequency is of particular interest because it is a quantity that can easily be obtained from the experimental data and furthermore gives a direct measure for the interaction energy $U$ : following Eq. (77), at the crossover frequency we have $\hbar \omega_{c}=U\left(r_{\omega_{c}}\right)$. In gen-

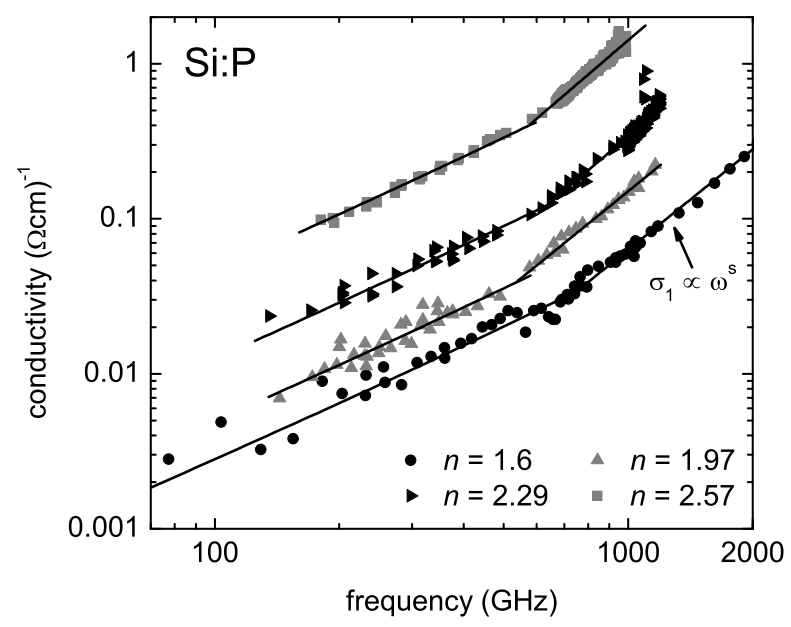

FIG. 4: Frequency-dependent conductivity of the four experimentally accessible Si:P samples. The respective phosphorus concentration is indicated in the graph in units of $10^{18} \mathrm{~cm}^{-3}$. The fits correspond to the super-linear and approximately quadratic behavior.

eral one expects the crossover frequency to decrease with increasing doping, approaching the MIT. This can be explained by the stronger screening of the Coulomb interaction due to the increase of the dielectric constant, as discussed in Sec.IVB below. Helgren et al ${ }^{\underline{16}}$ suggested that the crossover frequency scales with the doping concentration $\omega_{c} \propto\left|1-n / n_{c}\right|^{\beta}$ with $\beta \approx 1.65$; however, compared to those reports we observe a much weaker dependence; a power-law fit would yield an exponent $\beta \approx 0.21$. Note, however, that our values, but in particular the conductivity data and subsequently $\omega_{c}$ reported in Ref. 16 have considerably large error bars; hence we should not overinterpret this discrepancy.

Lee and Stutzmann $\frac{15}{}$ suggested that the width of the Coulomb gap affects the crossover energy. Combining temperature- and frequency-dependent data on Si:B they observe a relation $\hbar \omega_{c 2} \approx 2 \Delta$. Taking our sample with $n=2.57 \cdot 10^{18} \mathrm{~cm}^{-3}$ as an example, the temperaturedependent resistivity exhibits a kink around $4.6 \mathrm{~K}$ which indicates the transition from the Mott regime to the Efros-Shklovskii regime. From the fits in both limits [Eqs. (11) and (5)] we obtain $T_{\mathrm{Mott}}=2.25 \cdot 10^{6} \mathrm{~K}$ and $T_{\mathrm{ES}}=790 \mathrm{~K}$ which yields a Coulomb gap $\Delta=$ $1.15 \mathrm{meV}$, following Ref. 14. The corresponding frequency $280 \mathrm{GHz}$ is around half of the crossover frequency $\omega_{c} / 2 \pi=570 \mathrm{GHz}$, as suggested in Ref. 15 .

Whether this agreement indicates a close link between the width of the Coulomb gap and the crossover between the two regimes indicated in ac transport, however, remains an open question. One reason is that the validity of Eq. (7) is unclear for the important and experimentally relevant case of $U\left(r_{\omega}\right) \approx 2 \Delta$. Lee and Stutzmann proposed that the sharp crossover is controlled not by the average interaction strength $U$ as in Eq. (7), but instead by the Coulomb gap. In other words, it is not the 
mean Hartree energy between sites forming a resonant pair that is relevant, but the correlation energy $2 \Delta$. For this reason the single-particle gap measured by tunnelling is much larger than the renormalized Coulomb gap that governs transport. Helgren et al., 16 on the other hand, suggest that the Coulomb interaction determines the observed frequency-dependent crossover from ES- to Mottlike hopping conduction occurs. Based on recent computer simulations, Basylko et al. $\frac{17}{}$ also argue that the transition is driven by the Coulomb energy of sites forming resonant pairs and not by the width of the Coulomb gap. They calculated the number of sites outside the Coulomb gap relative to the total number of sites participating in the ac conductivity at a given frequency $\omega$, and found that within the frequency range of interest only sites inside the Coulomb gap contribute to the ac conductance, in accordance with our observation of a super-linear frequency dependence.

\section{B. Metal-Insulator Transition}

Besides the frequency-dependent conductivity, the dielectric constant $\epsilon_{1}(\omega, T)$ was measured for each Si crys-
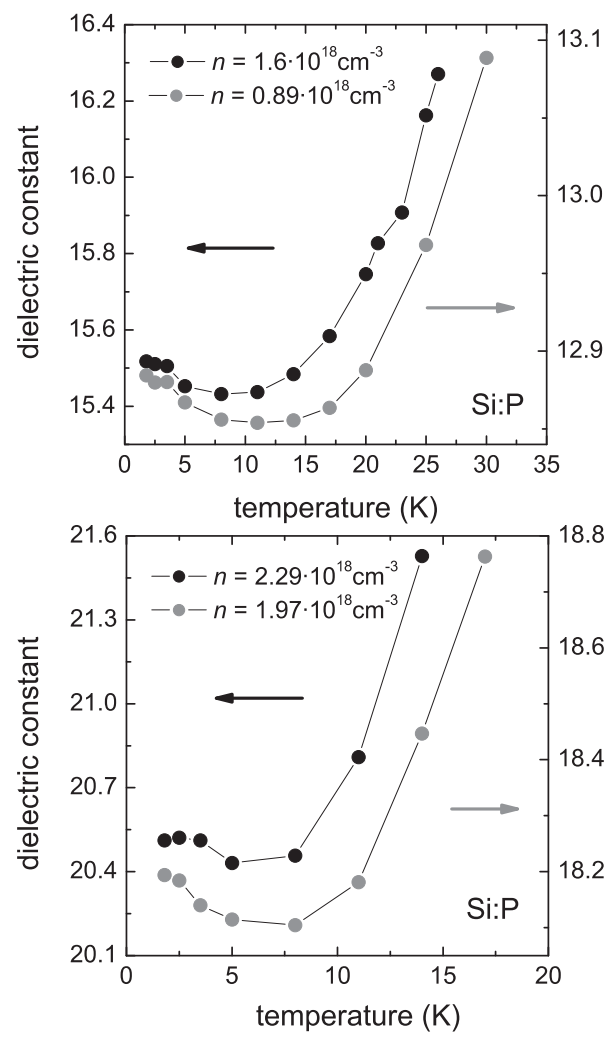

FIG. 5: Temperature dependence of the dielectric constant $\epsilon_{1}$ for Si:P samples with different phosphorus concentrations $n$ as indicated. The curves correspond to the left and right axes as indicated; also note the different temperature axes for the two frames. The data points are mean values averaged over the frequency range investigated.

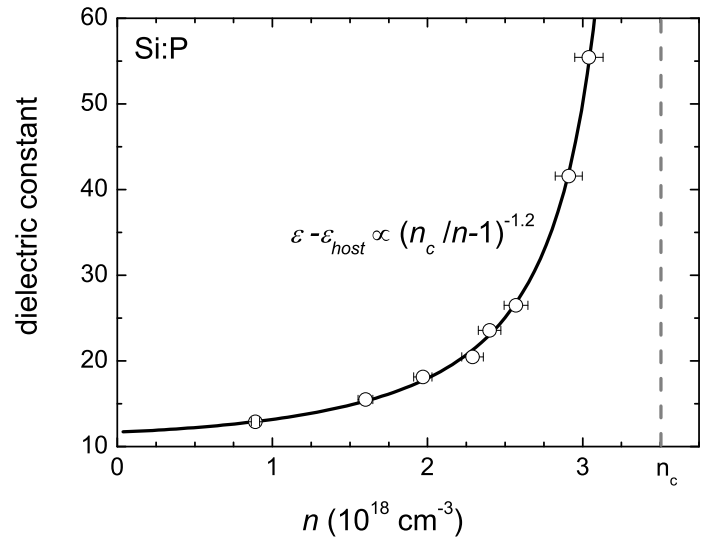

FIG. 6: Dielectric constant $\epsilon_{1}$ of Si:P as a function of phosphorus concentration measured at $T=1.8 \mathrm{~K}$. The solid line represents a fit by $\epsilon_{1}-\epsilon_{\text {host }} \propto\left(n_{c} / n-1\right)^{-\zeta^{\prime \prime}}$ with $\zeta^{\prime \prime}=1.2$.

tal of different phosphorus concentration. In the entire frequency range $30 \mathrm{GHz}<\omega / 2 \pi<3 \mathrm{THz}$, the dielectric constant $\epsilon_{1}(\omega)$ is basically frequency independent up to about $T=30 \mathrm{~K}$.

Surprisingly, as presented in Fig. 5. we find a nonmonotonic temperature dependence $\epsilon_{1}(T)$ with a pronounced minimum at low temperatures $T_{0}$ which might be an inherent property of the zero-phonon regime. Depending on the phosphorus concentration, the minimum decreases from approximately $T_{0}=11 \mathrm{~K}$ for $n=0.89 \cdot 10^{18} \mathrm{~cm}^{-3}$ to $T_{0}=5 \mathrm{~K}$ for samples with $n=2.91 \cdot 10^{18} \mathrm{~cm}^{-3}$. Within our range the concentration dependence of $T_{0}$ can be approximated by a linear behavior. Temperature-dependent measurements on undoped silicon do not exhibit such a minimum in the dielectric constant. 56 Also for samples with higher concentration there is no minimum evident in the observed temperature range; for these samples $T_{0}$ is very likely below the lowest temperature of $T=1.8 \mathrm{~K}$ that could be reached with our optical cryostat. It is tempting to suggest that the opening of the Hubbard gap $E_{2}$ leads to an additional contribution to the dielectric constant $\Delta \epsilon_{1} \propto E_{2}^{-2}$. Similar experiments on compensated samples could clarify this issue. 57

As can be seen in Fig. 5. for $T>T_{0}$ the dielectric constant increases with temperature because relaxation processes due to phonons become more likely. Note that the values of the dielectric constant for each temperature are obtained as the average of dozens of data points acquired in the frequency regime under inspection; they are very accurate (better than $0.5 \%$ ) and geometrical effects can be ruled out.

The low-temperature $\left(T \ll T_{0}\right)$ dielectric constant $\epsilon_{1}$ diverges when the phosphorus concentration increases towards the MIT as depicted in Fig. 6. In the present case it is more appropriate to subtract the lattice term $\epsilon_{\text {host }}=11.7$ of undoped silicon in order to gain direct insight to the critical behavior of the disordered localized system itself. Consequently Fig. [7 shows the concentra- 


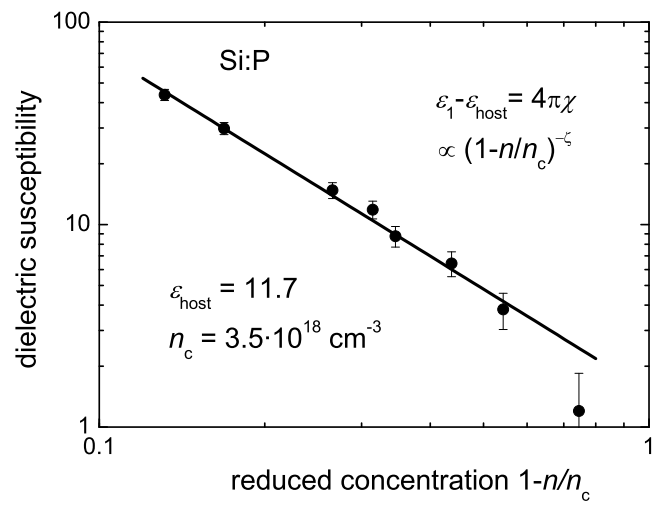

FIG. 7: Dielectric susceptibility $4 \pi \chi$ of $\mathrm{Si}: \mathrm{P}$ crystals versus the reduced doping concentration $1-n / n_{c}$ with $n_{c}=3.5$. $10^{18} \mathrm{~cm}^{-3}$ at $T=1.8 \mathrm{~K}$.

tion dependence of the dielectric susceptibility fitted by

$$
4 \pi \chi=\epsilon_{1}-\epsilon_{\text {host }} \propto\left(1-n / n_{c}\right)^{-\zeta} .
$$

The critical exponent $\zeta=1.68$ is in reasonable accord with previous reports $(\zeta=2.0$, Ref. 16$)$ taking the error bars of about $20 \%$ into account when comparing different samples. Significant deviations are observed for small phosphorus concentrations, i.e. further away from the MIT.

A resonable fit is obtained in the range $0.13>1-$ $n / n_{c}>0.5$. It should be pointed out that there is no clear indication of the opening of the Hubbard gap that was inferred to occur around $1-n / n_{c} \approx 0.8$ from transport measurements 27 We also note that for more heavily doped samples on the metallic side, the critical region of the critical exponent $\mu \approx 1$ of electrical dc conductivity, $\sigma_{d c} \propto\left|n-n_{c}\right|^{\mu}$, is much smaller, i.e. $1-n / n_{c} \approx 0.2 \underline{\underline{19}}$

There is some confusion in the literature because of an apparent "scaling" with the variable $n_{c} / n-1$, i.e. $\chi \propto\left|n_{c} / n-1\right|^{-\zeta^{\prime \prime}}$, extending to much larger reduced concentrations .54 .58 However, there is no theoretical justification for such a "scaling". For demonstration purpose only we note that the line shown in Fig. 66 is actually a fit with this function, yielding $\zeta^{\prime \prime}=1.2$ in strong contrast to $\zeta=1.68$, obtained from the fit to Eq. (12).

From temperature-dependent dc measurements Hornung et al.21 evaluated the concentration dependence of the Mott temperature $T_{\text {Mott }}$ and via Eq. (2) the scaling exponent $\nu=1.1$ for the correlation length. A scaling behavior is expected only very close to the MIT, nevertheless we can extract the relative concentration dependence of the localization length from the frequency-independent prefactor in Eq. (9), if we take the low-temperature value of the conductivity and dielectric constant. These results, plotted in Fig. 8, can be well described by a critical exponent of $\nu=0.87$ according to Eq. (10). Our findings are in excellent agreement with similar experiments for which $\nu=0.83$ was reported $\stackrel{16}{=}$ They fulfill the Harris criterion $\frac{59}{-} \nu>2 / d$, with $d$ the spatial dimension, which

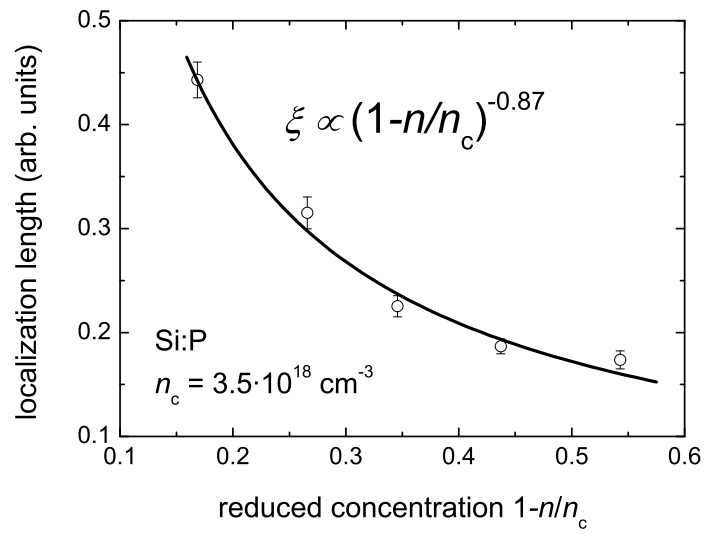

FIG. 8: Scaling behavior of the localization length in Si:P. The critical concentration is $n_{c}=3.5 \cdot 10^{18} \mathrm{~cm}^{-3}$.

implies that the disorder does not affect the critical behavior.

Castner and coworkers ${ }^{28.53}$ suggested a relation of the dielectric susceptibility to DOS and to the localization length

$$
\chi=e^{2} N\left(E_{F}\right) \xi^{2} .
$$

Thus the ratio of both critical exponents $\zeta / \nu$ is expected to be 2 which is perfectly confirmed by our measurements: $\zeta / \nu=1.97$. Looking at related systems, like doped germanium, indicates that the observed behavior is a general one, but also that the actual exponents depend on the doping range under consideration and the degree of compensation.

\section{CONCLUSIONS}

Our investigations of the electrodynamic properties of phosphorus doped silicon in a broad frequency range from microwaves up to the far infrared underline that electronic correlations between the localized states play an important role at low temperatures. The crossover in the optical conductivity from a linear to a quadratic behavior as predicted by Efros and Shklovskii is observed qualitatively; however, the simple model does not lead to a quantitative agreement. Our measurements show that the crossover energy $\hbar \omega_{c}$ is not solely determined by the interaction energy $U\left(r_{\omega}\right)$. Furthermore, the Coulomb gap in the density of electronic states explains the observation of a super-linear frequency dependence in the Coulomb glass regime. When approaching the MIT with increasing doping concentration, the dielectric constant and the localization length show critical behavior, in good agreement with theoretical predictions.

However, there remain open questions to be addressed in the future. Concerning the dielectric constant, there is the unclear origin of the minimum in the temperature dependence. As far as the conductivity is concerned, our results should be confirmed in comparable systems, like 
$\mathrm{Si}$ B or Si:As. Of superior importance is a detailed investigation of the influence of compensation: while the theory of Shklovskii and Efros was done for compensated semiconductors, most experiments (ours as well) have been performed on nominally uncompensated samples. Furthermore, studies in even larger parameter ranges are highly desired to close the gap not only in frequency but consequently also in concentration between the GHz results of Lee and Stutzmann 15 and the THz results of the present and other recent studies $\underline{\underline{16}}$ Here the concentration dependence of the frequency crossover is of particular interest.

Finally, it would be of interest to analyze the frequency-dependent conductivity at elevated temperature, i.e. when the zero-phonon regime is left. For $k_{B} T \approx \hbar \omega$ both photon-assisted and phonon-assisted processes are equally important. Although the data exist, suitable models are still lacking.

\section{Acknowledgments}

We would like to thank D. Schweitzer and W. Zulehner for providing the single-crystalline $\mathrm{Si}$ of various doping concentrations. The samples have been carefully prepared by G. Untereiner. We thank B. Gorshunov for advice on measurement techniques. A.W. Anajoh performed some of the dc experiments. We acknowledge the helpful discussions with G. Grüner, E. Helgren, M. Lee, E. Ritz and P. Wölfle.
* Present address: Kavli Institute of Nanoscience, Delft University of Technology, 2600 GA Delft, The Netherlands

$\dagger$ Electronic mail: dressel@pil.physik.uni-stuttgart.de URL: http://www.pi1.physik.uni-stuttgart.de

1 H. v. Löhneysen, Festkörperprobleme/Adv. Solid State Phys. 30, 95 (1990); Phil. Trans. R. Soc. London A 356, 139 (1998); Festkörperprobleme/Adv. Solid State Phys. 40, 143 (2000).

2 M. P. Sarachik, in: Metal-Insulator Transitions Revisited, edited by P. P. Edwards and C. N. Rao (Taylor and Francis, London, 1995), p. 79.

3 N.F. Mott, J. Non-Crystal. Solids 1, 1 (1968).

${ }^{4}$ N.F. Mott and E.A. Davis, Electronic Processes in NonCrystalline Materials, 2nd ed. (Oxford University Press, Oxford, 1979).

5 M. Pollak, Disc. Faraday Soc. 50, 13 (1970).

${ }^{6}$ G. Srinivasan, Phys. Rev. B 4, 2581 (1971).

7 A.L. Efros and B.I. Shklovskii, J. Phys. C 8, L49 (1975).

8 Electron-Electron Interaction in Disordered Systems, edited by A.L. Efros and M. Pollak (North-Holland, Amsterdam, 1984).

9 B.I. Shklovskii and A.L. Efros, Electronic Properties of Doped Semiconductors (Springer, Berlin, 1984).

10 P.W. Anderson, Comments in Solid State Phys. 2, 193 (1970).

11 J.H. Davies, P.A. Lee, and T.M. Rice, Phys. Rev. Lett. 49, 758 (1982); Phys. Rev. B 29, 4260 (1984).

12 B.I. Shklovskii and A.L. Efros, Zh. Eksp. Teor. Fiz. 81, 406 (1981) [Sov. Phys. JETP 54, 218 (1981)].

13 J.G. Massey and M. Lee, Phys. Rev. Lett. 75, 4266 (1995); ibid. 77, 3399 (1996); M. Lee, J.G. Massey, V.L. Nguyen, and B.I. Shklovskii, Phys. Rev. B 60, 1582 (1999).

14 J.G. Massey and M. Lee, Phys. Rev. B 62, R13270 (2000).

15 M. Lee and M.L. Stutzmann, Phys. Rev. Lett. 87, 56402 (2001).

16 E. Helgren, N.P. Armitage, and G. Grüner, Phys. Rev. Lett. 89, 246601 (2002); N.P. Armitage, E. Helgren, and G. Grüner, in: Concepts in Electron Correlation, edited by A.C. Hewson and V. Zlatic, (Kluwer 2003); E. Helgren, N.P. Armitage, G. Grüner, Phys. Rev. B 69, 014201 (2004).

17 S.A. Basylko, V.A. Onischouk, and A. Rosengren, Phys.
Rev. B 70, 024201 (2004).

18 M. Müller and L.B. Ioffe, Phys. Rev. Lett. 93, 256403 (2004).

19 H. Stupp, M. Hornung, M. Lakner, O. Madel, and H. v. Löhneysen, Phys. Rev. Lett. 71, 2634 (1993).

20 G.A. Thomas, Phil. Mag. B 52, 479 (1985).

21 M. Hornung and H. v. Löhneysen, Czech. J. Phys. 46, Suppl. S5, 2437 (1996); M. Hornung, M. Iqbal, S. Waffenschmidt, and H. v. Löhneysen, phys. stat. sol. (b) 218, 75 (2000).

22 H. v. Löhneysen and M. Welsch, Phys. Rev. B 44, 9045 (1991).

23 M. Lakner, H. v. Löhneysen, A. Langenfeld, and P. Wölfle, Phys. Rev. B 50, 17064 (1994).

24 M.A. Paalanen, T.F. Rosenbaum, G.A. Thomas, and R.N. Bhatt, Phys. Rev. Lett. 51, 1896 (1983).

25 S. Waffenschmidt, C. Pfleiderer, and H. v. Löhneysen, Phys. Rev. Lett. 83, 3005 (1999).

26 S. Bogdanovich, M.P. Sarachik, and R.N. Bhatt, Phys. Rev. Lett. 82, 137 (1999) and references therein.

27 X. Liu, A. Sidorenko, S. Wagner, P. Ziegler, and H.v. Löhneysen, Phys. Rev. Lett. 77, 3395 (1996)

28 T. G. Castner, in: Hopping Transport in Solids, edited by M. Pollak and B.I. Shklovskii (North-Holland, Amsterdam, 1991).

29 M. Dressel and G. Grüner, Electrodynamics of Solids (Cambridge University Press, Cambridge, 2002).

30 D. Belitz and T.R. Kirkpatrick, Rev. Mod. Phys. 66, 261 (1994).

31 S.L. Sondhi, S.M. Girvin, J.P. Carini, and D. Shahar, Rev. Mod. Phys. 69, 315 (1997).

32 P.A. Lee and T.V. Ramakrishnan, Rev. Mod. Phys. 57, 287 (1985).

33 P.W. Anderson, Phys. Rev. 109, 1492 (1958); E. Abrahams, P.W. Anderson, D.C. Licciardello, and T.V. Ramakrishnan, Phys. Rev. Lett. 42, 673 (1979).

34 F.J. Wegner, Z. Physik B 25, 327 (1976).

35 D. Stauffer, Phys. Rep. 54, 1 (1979).

36 I. Webman, J. Jortner, and M.H. Cohen, Phys. Rev. B 11, 2885 (1975).

37 A.L. Efros and B.I. Shklovskii, phys. stat. sol. (b) 76, 475 (1976). 
38 D.J. Bergman and Y. Imry, Phys. Rev. Lett. 39, 1222 (1977); D.J. Bergman, Phys. Rep. 43, 377 (1978); D.J. Bergman, Phys. Rev. Lett. 44, 1285 (1980).

39 D.M. Grannan, J.C. Garland, and D.B. Tanner, Phys. Rev. Lett. 46, 375 (1981).

${ }^{40}$ Y. Song, T.W. Noh, S.-I. Lee, and J.R. Gaines, Phys. Rev. B 33, 904 (1986).

41 M.T. Clarkson and S.I. Smedley, Phys. Rev. A 37, 2070 (1988); M.T. Clarkson, Phys. Rev. A 37, 2079 (1988).

42 F. Brouers, J.P. Clerc, and G. Giraud, Phys. Rev. B 44, 5299 (1991); F. Brouers, J.P. Clerc, G. Giraud, J.M. Laugier, and Z. Randriamantany, Phys. Rev. B 47, 666 (1993).

43 Y. Imry, Y. Gefen, and D. Bergman, in: Anderson localization, edited by Y. Nagaoka and H. Fukuyama (SpringerVerlag, Berlin 1982), p. 138.

44 W.L. McMillan, Phys. Rev. B 24, 2739 (1981).

45 One single crystal was grown by Société Générale Métallurgique de Hoboken and provided by D. Schweitzer. The other set stems from Wacker Chemitronic and was provided by W. Zulehner; it was previously characterized in Karlsruhe using various techniques. ${ }^{23,46}$

46 A. Gaymann, H.P. Geserich, and H. v. Löhneysen, Phys. Rev. Lett. 71, 3681 (1993); Phys. Rev. B 52, 16486 (1995).

47 Y. Outuka, F. Komori, Y. Monden, S. Kobayashi, and W. Sasaki, Solid State Commun. 36, 827 (1980).

48 W.R. Thurber, R.L. Mattis, Y.L. Liu, and J.J. Filliben, J. Electrochem. Soc. 127, 1807 (1980).

49 M. Hornung, Diploma Thesis, Karlsruhe 1993.

${ }^{50}$ G. Kozlov, A. Volkov, in G. Grüner: Millimeter and sub- millimeter wave spectroscopy of solids (Springer, Heidelberg, 1998).

51 Details of the data analysis will be given elsewhere. B. Gorshunov, J. van Slageren, and M. Dressel (unpublished).

52 R.J. Deri and T.G. Castner, Phys. Rev. Lett. 57, 134 (1986); T.G. Castner, W.N. Shafarman, R.J. Deri, and J.S. Brooks, J. Phys. C 19, L491 (1986); T.G. Castner and R.J. Deri, in: Disordered Semiconductors (Plenum Press, New York, 1987).

53 M. Migliuolo and T.G. Castner, Solid State Commun. 67, 863 (1988).

54 H.F. Hess, K. DeConde, T.F. Rosenbaum, and G.A. Thomas, Phys. Rev. B 25, 5578 (1982).

${ }^{55}$ Indications of a mimimum in the temperature dependence of the dielectric constant can be found (without discussion) in the $3 \mathrm{kHz}$ data of Tan and Castner ${ }^{56}$ on Si:P with $n=1.43 \cdot 10^{18} \mathrm{~cm}^{-3}$. No minimum is found for $n \leq 5.1 \cdot 10^{17} \mathrm{~cm}^{-3}$.

${ }^{56}$ H. S. Tan and T.G. Castner, Phys. Rev. B 23, 3983 (1981).

57 There is one report of optical measurements which is supposed to indicate the formation of the Hubbard gap in very low-doped Si; P. Norton, Phys. Rev. Lett. 37, 164 (1976).

58 M. Capizzi, G.A. Thomas, F. DeRosa, R.N. Bhatt, and T.M. Rice, Phys. Rev. Lett. 44, 1019 (1980).

${ }^{59}$ A.B. Harris, J. Phys. C 7, 1671 (1974).

60 A.N. Ionov, I.S. Shlimak, M.N. Matveev, Solid State Commun. 47, 763 (1983).

61 M. Watanabe, K.M. Itoh, Y. Ootuka, E.E. Haller, Phys. Rev. B 62, 2255 (2000). 